\title{
Non-Rotating Black Holes, Dark Matter and Dark Energy in a Unifying Theory
}

\author{
Antonio Holdefer \\ IFG, Neropolis, Goias, Brasil \\ Email: holdefer@gmail.com
}

How to cite this paper: Holdefer, A. (2021) Non-Rotating Black Holes, Dark Matter and Dark Energy in a Unifying Theory. Journal of Applied Mathematics and Physics, 9, 1560-1582.

https://doi.org/10.4236/jamp.2021.97107

Received: June 20, 2021

Accepted: July 23, 2021

Published: July 26, 2021

Copyright (c) 2021 by author(s) and Scientific Research Publishing Inc. This work is licensed under the Creative Commons Attribution International License (CC BY 4.0).

http://creativecommons.org/licenses/by/4.0/ (c) (i) Open Access

\begin{abstract}
The theory here developed, makes use of the decomposition of matter (mass) in different spatial frequencies $k^{2} s$ using spatial Fourier transforms, and the posterior use of modified inverse Fourier transforms to construct an accurate description of the classical Newtonian gravitational field. Introducing the concept of quantization of the spatial frequency $k$, which means allowing only discrete values, such as $k_{m}, 2 k_{m}, 3 k_{m}$, leads to the appearance of extra gravitational force regions that occur at distances equally spaced apart in $2 \pi / k_{m}$. These areas of extra gravitational force decrease inscribed in an inverse of the distance envelope $(1 / r)$. The value of $2 \pi / k_{m}$ can be adjusted to be of the order of kiloparsec $(\mathrm{kpc})$, being this way a plausible explanation for the effect of the dark matter since this causes practically flat rotation curves for most of the galaxies. As these regions of extra gravitational force also have adjacent areas of negative values (repulsive gravitational force), it is possible to show that any mass placed in the gravitational field far from the galaxy center will acquire, on average, a null acceleration, thereby remains the "light push," or in other words, the "mean luminosity density" between galaxies as an explanation for the accelerating expansion of the universe, today being considered mainly due to dark energy. Along with the article, it is showed that the effect of light push is sufficient to explain the expansion of the universe. The present work also explains the nonlinear behavior of gravitational fields near massive objects such as blackholes, not contradicting the theory of general relativity, instead giving a complementary description of how black holes work, even describing the gravitational field internally to it, which is not available in the GR theory.
\end{abstract}

\section{Keywords}

Black Holes, Dark Matter, Dark Energy, Motion Energy, Accelerating Expansion of the Universe 


\section{Introduction}

The theory of general relativity $G R[1]$ has been proved to be accurate in most cases, any attempt to complement it must be taken carefully.

Despite its immense success, $G R$ theory lacks in explaining the behavior of the gravity inside black holes [2]. Besides, the calculation of the acceleration of gravity outside a black hole or any other massive celestial object having a strong gravitational pull is the subject of numerically solving complex nonlinear equations [3]. Moreover, finally, there is a bridge that has yet to be built between $G R$ and quantum mechanics [4].

The present theory has the merit and the novelty of helping to explain or solve the three points above, besides offering a simple computational way to calculate gravity inside and outside black holes, or in any other massive object, using Fourier transforms, such as explained later.

As a start point for the theory of black holes, the author considers the existence of two kinds of energy sources for the gravitational field: The rest mass, which converts into energy by $E=M c^{2}$ and the energy due the motion of particles.

For calculations are used the Fourier transform, widely used in quantum mechanics for representing the wave function in the position or momentum space, among many other uses [5].

Regarding dark matter and dark energy, both have been the subject of discussion for a long time [6], with some works done in an attempt to relate the two effects [7].

Based on the work discussed above, it is possible to consider, by hypothesis, the possibility of the quantization of the spatial frequency $k$, that is, only occurring at fixed intervals, such as $k_{m}, 2 k_{m}, 3 k_{m}$, where $k_{m}$ stands for "minimum spatial frequency".

The proposed quantization, when applied in the inverse Fourier transform, reveals areas of extra gravitational force spaced at $2 \pi / k_{m}$, which fall as inscribed in an inverse distance envelope $(1 / r)$. Therefore, choosing the correct value of $2 \pi / k_{m}$ in the range of kiloparsecs ( $\mathrm{kpc}$ ) is sufficient to explain the practically flat rotation curves of most galaxies [8]. Also, the appearance of adjacent regions of negative gravitational force due to the proposed quantization is also verified. Areas of negative gravitational force cancel, on average, the effect of areas of positive gravitational force, which means that an object traveling between galaxies experiences, on average, a null gravitational effect.

The null average effect of gravity between galaxies allows us to demonstrate that the mean luminosity density of the universe is a sufficient factor to explain the repulsive force responsible for the accelerating expansion of the universe.

By now, it is not clear what causes the universe to expand, puzzling the scientists even more since the discovery that the expansion is in fact accelerating [9] [10].

The repulsive force of light as responsible for the accelerating expansion of the universe is mostly unconsidered due to its small magnitude compared to gravi- 
tational effects, being necessary the elaboration of alternative concepts such as negative vacuum pressure or dark energy, among others [11].

However, considering the average annulment of the force of gravity between galaxies, it is clear that luminosity could be a predominant factor and the only one necessary to explain the expansion of the universe. The physical model developed in the last part of the present paper has yielded results that agree very well with values that are within the current acceptable limits for the Hubble constant $\approx 67-73 \mathrm{~km} \cdot \mathrm{s}^{-1} \cdot \mathrm{Mpc}^{-1}$, obtained respectively by [12] and [13].

The present model excludes the existence of non-baryonic matter, or the existence of dark matter or dark energy, further concludes that the expansion of the universe follows a coasting universe model, expanding forever, never contracting.

\section{Decomposition of Matter in k-Space}

The k-space or reciprocal space is the spatial frequency domain of a spatial Fourier transform, as shown in Equation (1). It is possible to take a distribution of mass in position space, and through the use of a spatial Fourier transform to decompose it in different spatial frequencies.

$$
D(k)=\int_{-\infty}^{\infty} D(x) \cdot \mathrm{e}^{-i x k} \mathrm{~d} x
$$

As our object of study are mostly radially symmetric spherical bodies, such as stars, black holes, and so on, it is showed, using spherical coordinates, that the Fourier transform for such a case can be simplified as in Equation (2) [14].

$$
D(k)=\int_{0}^{R} D(r) \cdot 4 \pi r^{2} \cdot \frac{\sin (k r)}{k r} \mathrm{~d} r
$$

where $r$ is the radial coordinate, and $k$ is the spatial frequency in k-space, which unit is $\mathrm{m}^{-1}$. For a spherical object of mass $M$, radius $R$, and volume $V$, the radial density $D(r)$ is as shown in Equation (3).

$$
D(r)=\frac{M}{V}=\frac{3 M}{4 \pi R^{3}}
$$

Substituting Equation (3) in Equation (2) and resolving for $D(k)$, we get

$$
D(k)=\frac{3 M}{R^{3}} \frac{\sin (R k)-R k \cos (R k)}{k^{3}}
$$

where $R$ is the radius of the spherical object, $k$ is the spatial frequency and $M$ is the mass of the object. The function $D(k)$ has unit $\mathrm{kg}$ and can be understood as being the decomposition of the mass $M$ in different spatial frequencies $k s$. Equation (4) is plotted in Figure 1.

From Figure 1, we see that the decomposition of an object of mass $M$, with uniform density and radius $R$ in k-space, has a maximum value of $M$ and a "bandwidth" inversely proportional to the radius $R$. However, we can observe the existence of negative values, which does not necessarily mean negative mass, which has no physical significance. Instead negatives values in k-space are understood as spatial frequencies shifted $180^{\circ}$ in phase, being necessary for the correct reconstruction 


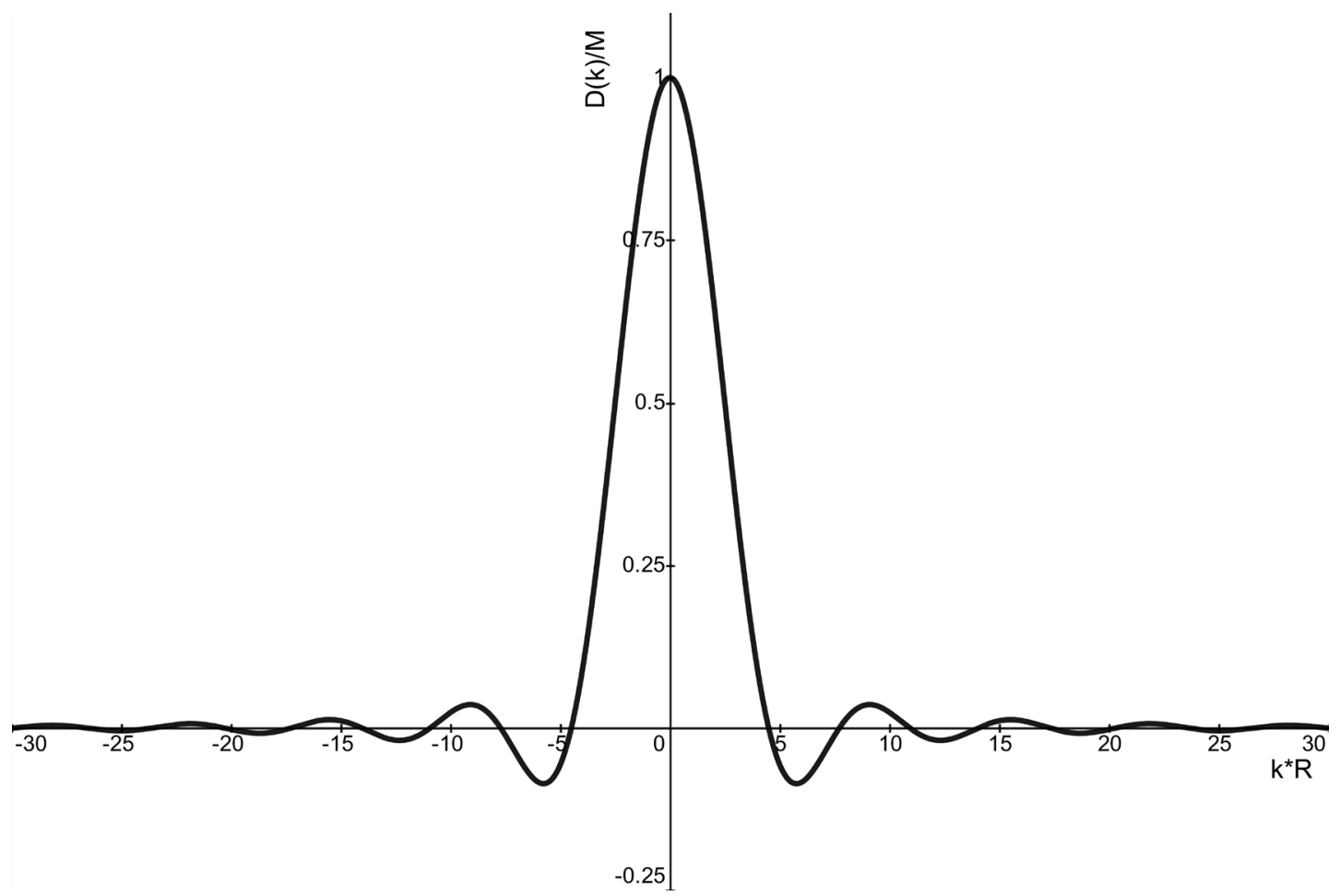

Figure 1. Decomposition in k-space of an object with mass $M$, radius $R$, and uniform density.

of the object after the application of the inverse Fourier transform, as will be explained below.

\section{Reconstruction of the Gravitational Potential}

To facilitate the understanding of topics to be discussed later, we will normalize the function $D(k)$, that is, divide it by $M$ and introduce the function $F(k)$ which is simply $D(k) / M$.

The inverse Fourier transform associated with the Fourier transform of Equation (2) is defined as

$$
D(r)=\frac{1}{(2 \pi)^{3}} \int_{0}^{\infty} M \cdot F(k) \cdot 4 \pi k^{2} \cdot \frac{\sin (k r)}{k r} \mathrm{~d} k
$$

Upon inspection, it is verified that the right transformation for the classical Newtonian gravitational potential is given by Equation (6).

$$
U(r)=\frac{2}{\pi} \int_{0}^{\infty} G M \cdot F(k) \cdot \frac{\sin (k r)}{k r} \mathrm{~d} k
$$

Which gives the correct value for the classical Newtonian gravitational potential, of the form $G M / r$, where $G$ is the gravitational constant.

Plotting the Equation (6) along with its negative derivative with respect to $r$ $(a(r)=-\mathrm{d} U(r) / \mathrm{d} r)$, which is the force per unit mass i.e., gravitational acceleration, we obtain as shown in Figure 2 the correct value for the gravitational potential and force per unit mass (gravitational acceleration) in the classical Newtonian form. As a further verification of the presented method, in Figure 3 


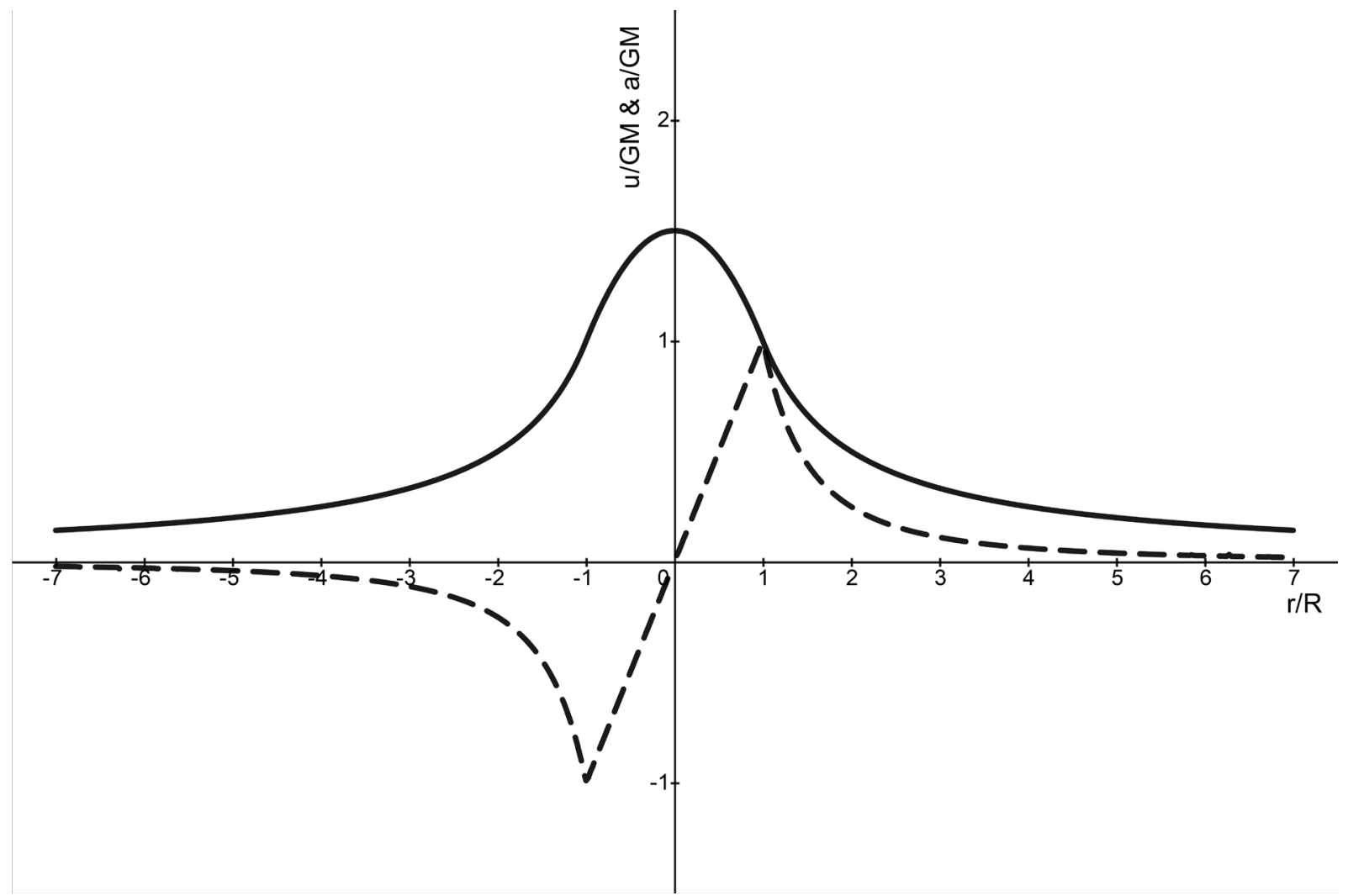

Figure 2. Gravitational potential (Solid line) and force per unit mass (Dashed line) obtained from Equation (6) for a solid sphere.

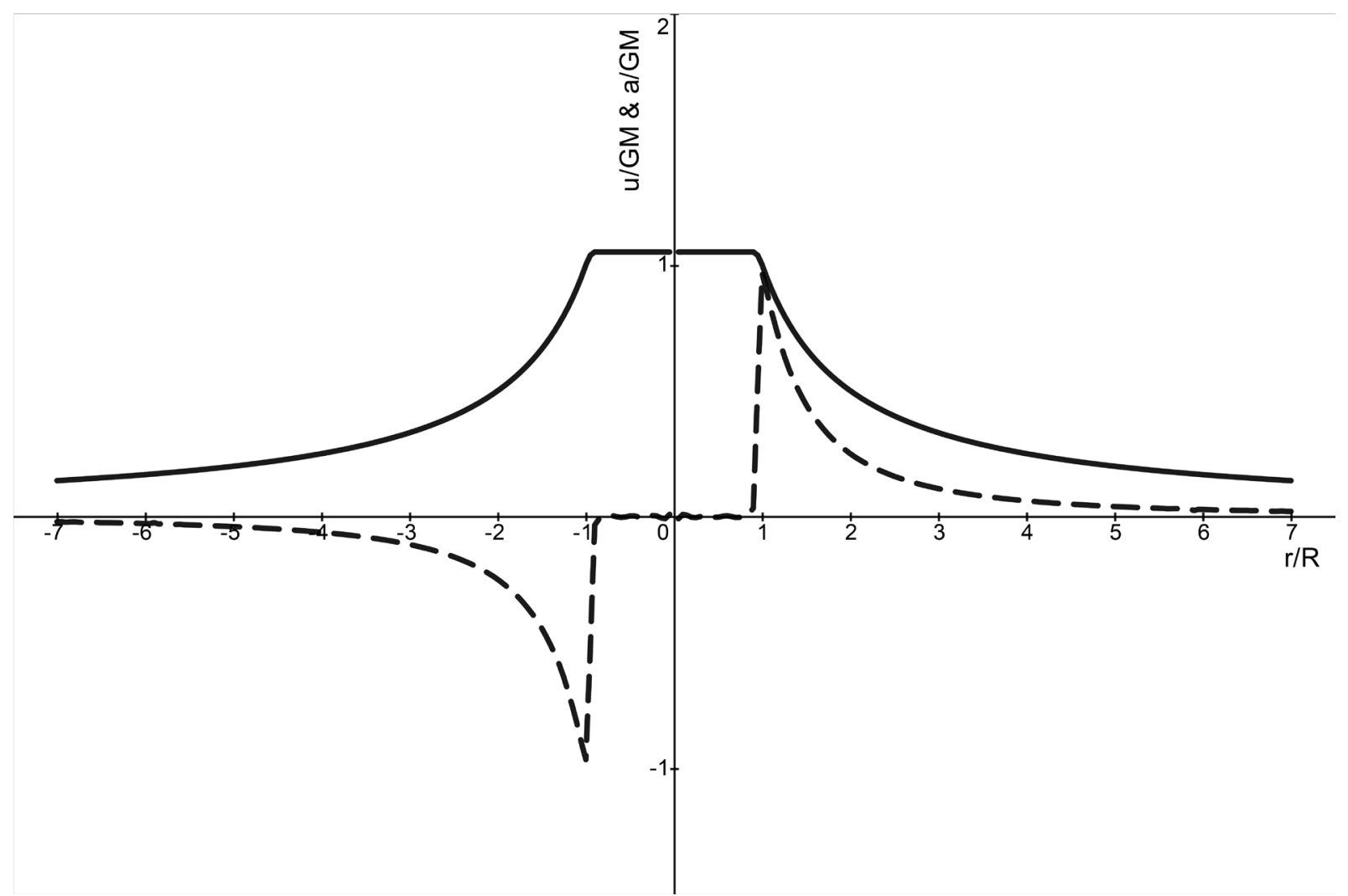

Figure 3. Gravitational potential (Solid line) and force per unit mass (Dashed line) for a hollow sphere. 
is plotted the value of the gravitational potential for a hollow sphere using the pair of direct and inverse Fourier transforms given respectively by the Equations (2) and (6) and using Equation (3) considering the volume $V$ as the volume of the outer shell. Where the integration limits for the Equation (2) are taken between $0.9 R$ and $R$. The respective value of its negative derivative (acceleration) is also showed in the dashed line.

Such previous examples prove the validity of the adopted method. Also, other validated cases are not shown here.

The same concept could be expanded to objects that do not necessarily have radial symmetry, for that it is enough to use the correct pairs of Fourier transforms for such cases. These results indicate that any radial distribution of matter can be decomposed in k-space, using Equation (2), and by using Equation (6) to reconstruct the corresponding classical Newtonian gravitational potential, which by the negative value of its differentiation in space results in force per unit mass (acceleration).

\section{Motion Energy with Increasing in Mass Density}

In the relativity theory [15], the total energy $E$ of an object is

$$
E^{2}=(p c)^{2}+\left(M c^{2}\right)^{2}
$$

With $M c^{2}$ being the rest mass energy (from now on the mass $M$ is also considered the rest mass) and its "motion energy" given by the product of its momentum and the speed of light $c$. Based on that, the theory presented here assumes that the use of Equation (6) only considers the rest mass $M$ or in a similar way, the "rest energy" $M c^{2}$ to correctly calculate the classical Newtonian gravitational potential.

However, the motion energy of an object is not only due its translational motion, being also contained in the form of quantum motion of its constituent particles.

Such phenomenon is described in the well-known "Uncertainty Principle" [16], shown in Equation (8) and which essentially states that the greater the certainty in determining the momentum of the particle, the less certainty in its position and vice versa.

$$
\Delta p \geq \frac{\hbar}{2 \Delta x}
$$

where $\hbar$ is the reduced Planck constant.

This principle can be used to estimate the average values for the motion energy of a quantum particle, which is inversely proportional to the dimension occupied by the particle $\Delta x$.

It is assumed here that the increase in mass density will act in reducing the radius of the particles (more particles packed together) and consequently increasing the motion energy of its constituent particles. This can be clearly seen in Figure 1, where the "bandwidth" is proportional to $1 / R$, meaning that as the ra- 
dius of the spherical object decreases the amount of energy in the "momentum space" $k$ spreads out, having consequently more energy in the form of quantum motion.

\section{Gravitational Potential due the Motion Energy of Quantum Particles}

The spatial frequency $k$ is directly related to the momentum of a particle through the De Broglie relation $h k$, where $h$ is the Planck constant. As the Fourier transform indicated in Equation (2) is a 3D transform, the same is valid for the inverse transform. So, integrating the function all over the $3 \mathrm{D}$ space of the spatial frequency $k$, we obtain the expression in Equation (9), which indicates the gravitational potential due to the motion energy.

$$
U_{M}(r) \propto \int_{0}^{\infty} \frac{G^{3}}{c^{4}} M^{3} F(k) \cdot k^{2} \frac{\sin (k r)}{k r} \mathrm{~d} k
$$

where physical constants and extra terms were adjusted in order to give the same dimension as in Equation (6), since the unit for $k$ is $\mathrm{m}^{-1}$.

As the Equation (7) indicates that the rest mass energy and the momentum energy are perpendicular to each other we can suppose that the angle total energy $E$ is shifted in arctan (Momentum energy/Total Energy) in relation to the rest mass. This all can be summarized in the Equation (10).

$$
\begin{aligned}
U_{T}(r)= & \frac{\pi}{2} \int_{0}^{\infty} F(k) \cdot \sqrt{(G M)^{2}+\left(2 \frac{G^{3}}{c^{4}} M^{3} \cdot k^{2}\right)^{2}} \\
& \frac{\sin \left(k r-\arctan \left(2 \frac{G^{2}}{c^{4}} M^{2} \cdot k^{2}\right)\right)}{k r-\arctan \left(2 \frac{G^{2}}{c^{4}} M^{2} \cdot k^{2}\right)} \mathrm{d} k
\end{aligned}
$$

where $U_{T}$ is the complete solution for the gravitational potential. The terms in the square root are respectively proportional to the rest mass energy and motion energy and the term inside the arctan function represents the ratio between them. The values 2 multiplying the motion energy were chosen in order to adjust the equation to the $G R$ theory as seen later. The Equation (10) is reduced to the classical gravitational potential as in Equation (6) in the limit $R \gg R_{S}$, where $R_{S}$ is the Schwarzschild radius.

\section{Non-Rotating Black Holes}

Making use of the Equation (10), we are able to plot for the condition for the formation of a black hole $R=R_{S}$, being $R_{S}=2 G M / c^{2}$.

For comparison we use the relativistic gravitational potential as obtained by [17] and indicated in Equation (11).

$$
U_{T}(r)=-\frac{c^{2}}{2} \ln \left(1-\frac{2 G M}{r c^{2}}\right)
$$


By analyzing Figure 4, which is the plotting of Equation (18), we realize that the gravitational force only goes to infinity at the border of a black hole.

Which gives a pretty good agreement and besides that offers an understanding of how the gravitational field behaves internally to the massive body, showing that the gravitational potential only tends to infinity in the border of the black hole.

Such procedure can be used for calculation of the gravitational field near massive bodies approaching the Schwarzschild limit.

An important question that can be raised throughout this topic is how the mass contained in an object can deflect the space-time fabric to exert a force at the distance on some other object? As an answer, we can infer that any object that has mass has constituent particles that are always in motion, and that movement would be transmitted to the space-time fabric in a way to combine with the other vibrations originated by other particles building up a resulting total deflection of the space-time fabric in all internal and external regions of such object.

The only difference between the deflection caused by rest mass and the motion energy of these particles would be the way they "move", causing different effects on the space-time fabric that would later be transmitted to all regions of the space.

Up to here, we have worked with gravitational effects more evident over small

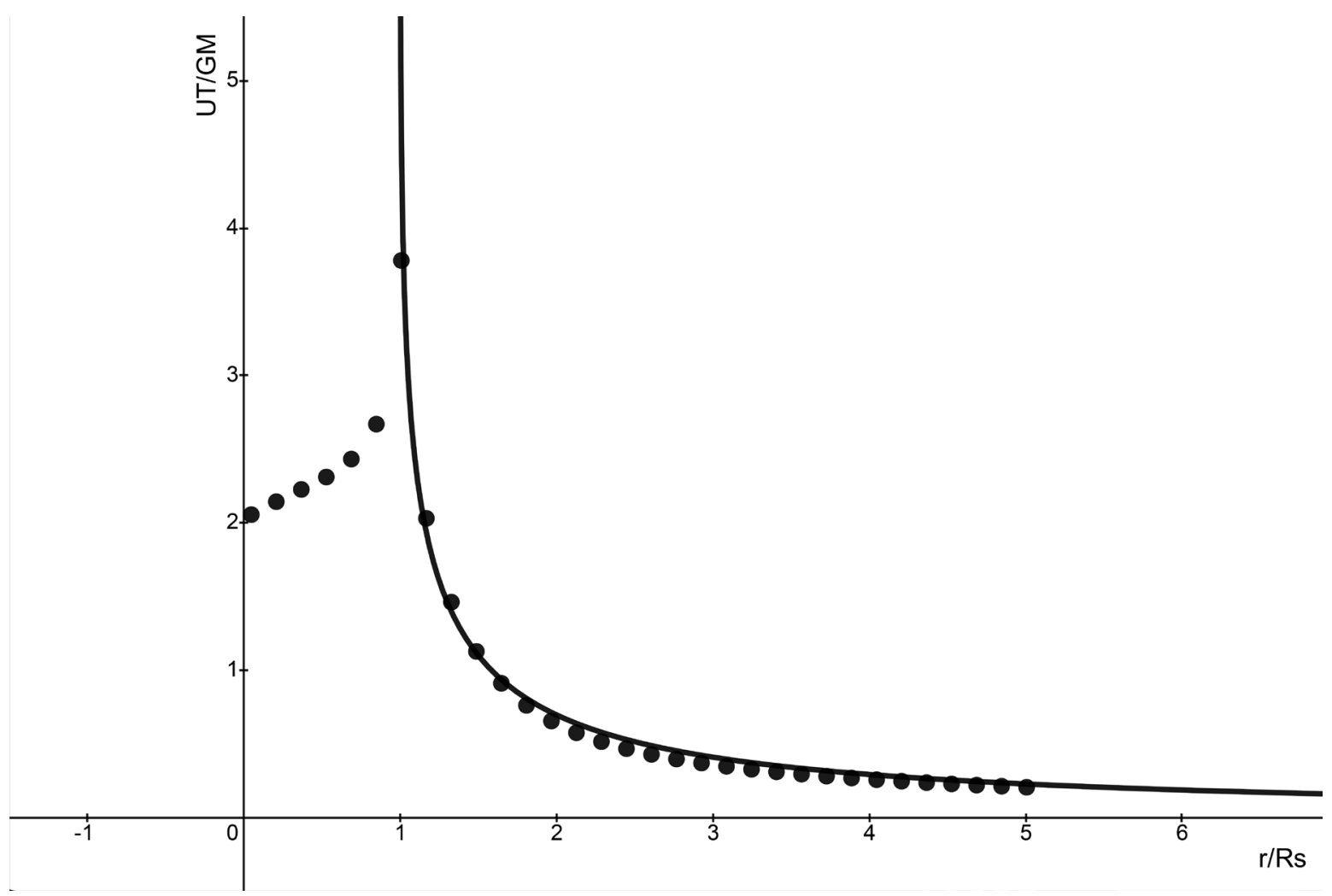

Figure 4. Gravitational potential field for a black hole. Numerical solution using Equation (10) (Circle), and the relativistic gravitational potential using Equation (11) (Solid line) for comparison. 
distances on cosmological scales $\ll 1 \mathrm{kpc}$, contained in smaller dimensions than those of our solar system. Since the extra gravitational effect of black holes seems more obvious in their surroundings, it became asymptotically more similar to the classic Newtonian model for greater distances, since the extra effect due to the motion energy of the quantum particles decays more abruptly as distance increases.

However, for larger cosmological scales in the order of $\mathrm{kpc}$, we verify the existence of the phenomenon known as dark matter, and on even larger scales (intergalactic medium) we verify the existence of the phenomenon of dark energy.

In the next topic, we will demonstrate that both dark matter and dark energy can be caused by the existence of the quantization of the spatial frequency $k$, which can be understood as a limitation of the matter moving at all values of spatial frequency $k$, or a limitation of the space-time field of being excited in all spatial frequencies, with such excitations only occurring in steps.

We must clarify here that the quantization of the spatial frequency does not affect the results obtained in the present topic, since it only has effects on a scale much larger than that of our solar system.

\section{Quantization of the Spatial Frequency $k$}

We here postulate the existence of a minimum value for the spatial frequency $k$, being the other values multiples of this minimum value. Therefore, the spatial frequency can only acquire discrete values, such as $k_{m}, 2 k_{m}, 3 k_{m}$, and so on.

This leads to a discrete representation of the integral in Equation (6), with an increment of $k_{m}$, as indicated in Equation (12).

$$
\begin{aligned}
& U(r)=\frac{2}{\pi} \sum_{n=1}^{\infty} G M \cdot F\left(n \cdot k_{m}\right) \frac{\sin \left(n \cdot k_{m} \cdot r\right)}{n \cdot k_{m} \cdot r} k_{m} \\
& a(r)=-\frac{\mathrm{d}}{\mathrm{d} r} U(r)
\end{aligned}
$$

The value of $k_{m}$ should be considered as very small, in such a way that the value of $2 \pi / k_{m}$ is on the galaxies size scale $(\mathrm{kpc})$. Therefore, the celestial objects considered in this work are galaxies in general, where the effects of the quantization of the spatial frequency are evident.

\subsection{Dark Matter}

To demonstrate how the quantization of the spatial frequency $k$ allows us to deduce the effect of dark matter, we will take the example of the Milky Way.

The distribution of mass in the bulge of the Milky Way $D_{b}(r)$ can be modeled according to Equation (13) [18].

$$
D_{b}(r)=\frac{3 \cdot M \cdot b^{2}}{4 \cdot \pi \cdot\left(r^{2}+b^{2}\right)^{\frac{5}{2}}}
$$

With $M=9.5 \times 10^{9} M_{\odot}$ and $b=1.9 \mathrm{kpc}$. This equation can be approximated using a bell curve, as indicated in Equation (14). 


$$
D_{b}(r)=\frac{M \cdot \mathrm{e}^{-\left(\frac{x}{b}\right)^{2}}}{\pi^{\frac{3}{2}} \cdot b^{3}}
$$

In Figure 5, we can see that the two curves are similar, and the use of one or the other is indifferent to our goal of showing the effect of the quantization of the spatial frequency $k$ in the behavior of the gravitational force. However, we will choose to use the bell curve because it is easier to solve analytically.

After the application of Equation (14) in Equation (2), with $R \rightarrow \infty$, we obtain the mass distribution in $k$-space, also a bell curve, in terms of the spatial frequency $k$, as shown in Equation (15).

$$
\begin{aligned}
& D_{b}(k)=M \cdot \mathrm{e}^{\frac{-(k \cdot b)^{2}}{4}} \\
& F_{b}(k)=\frac{D_{b}(k)}{M}=\mathrm{e}^{\frac{-(k \cdot b)^{2}}{4}}
\end{aligned}
$$

Applying this value in Equation 12, with $k_{m}=1 \mathrm{kpc}^{-1}$ and comparing the effect with the classic Newtonian force, we get Figure 6. From Figure 6, we can see that the effect of the quantization of the spatial frequency creates regions of extra gravitational force that decrease inscribed in a $1 / r$ envelope. This phenomenon is like that found in signal analysis, more specifically in the sampling theory, where the discretization of the signal in one domain, produces extra spectrums in the other domain [19].

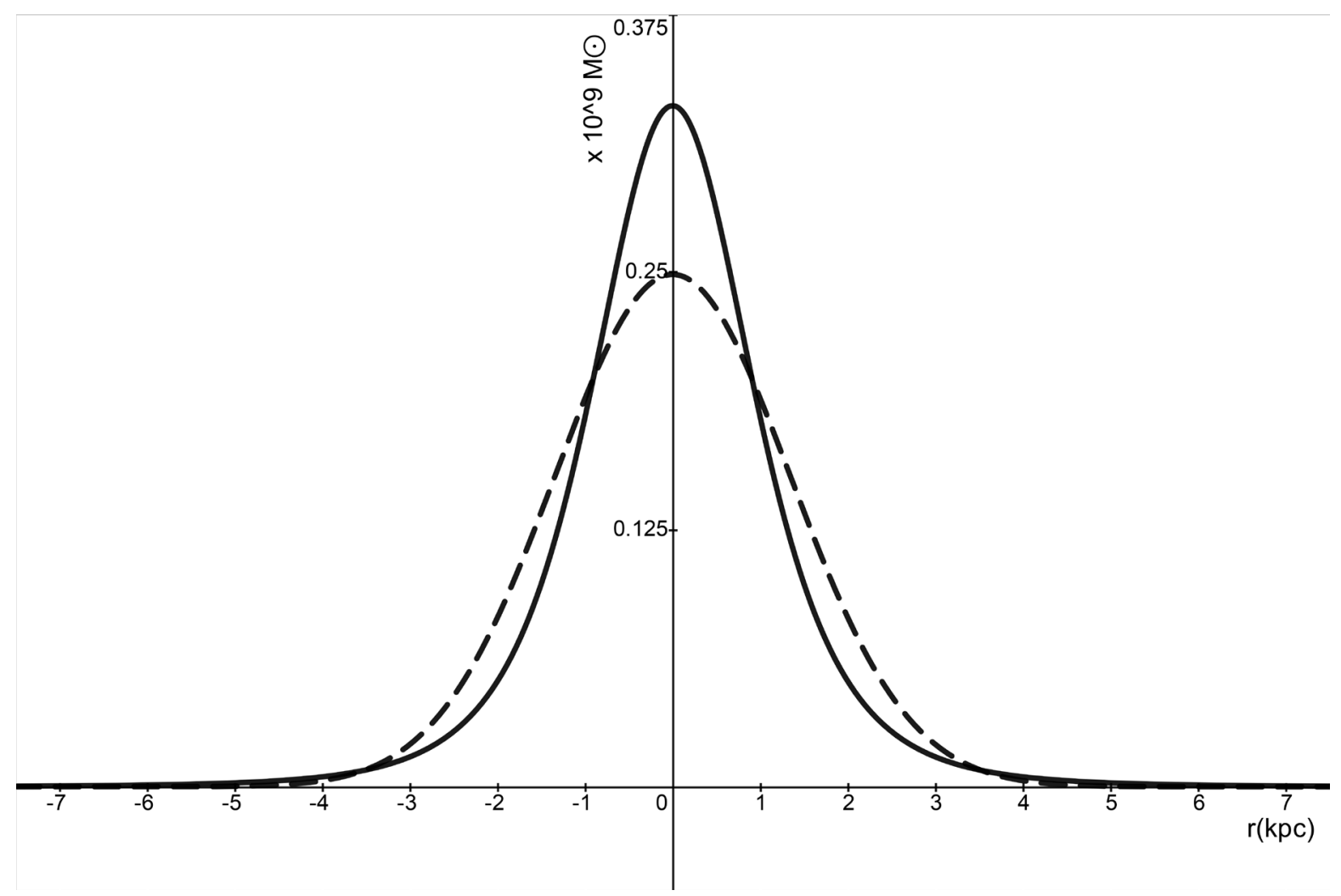

Figure 5. Equation (13) (Solid line) and Equation (14) (dashed line) for comparison. 


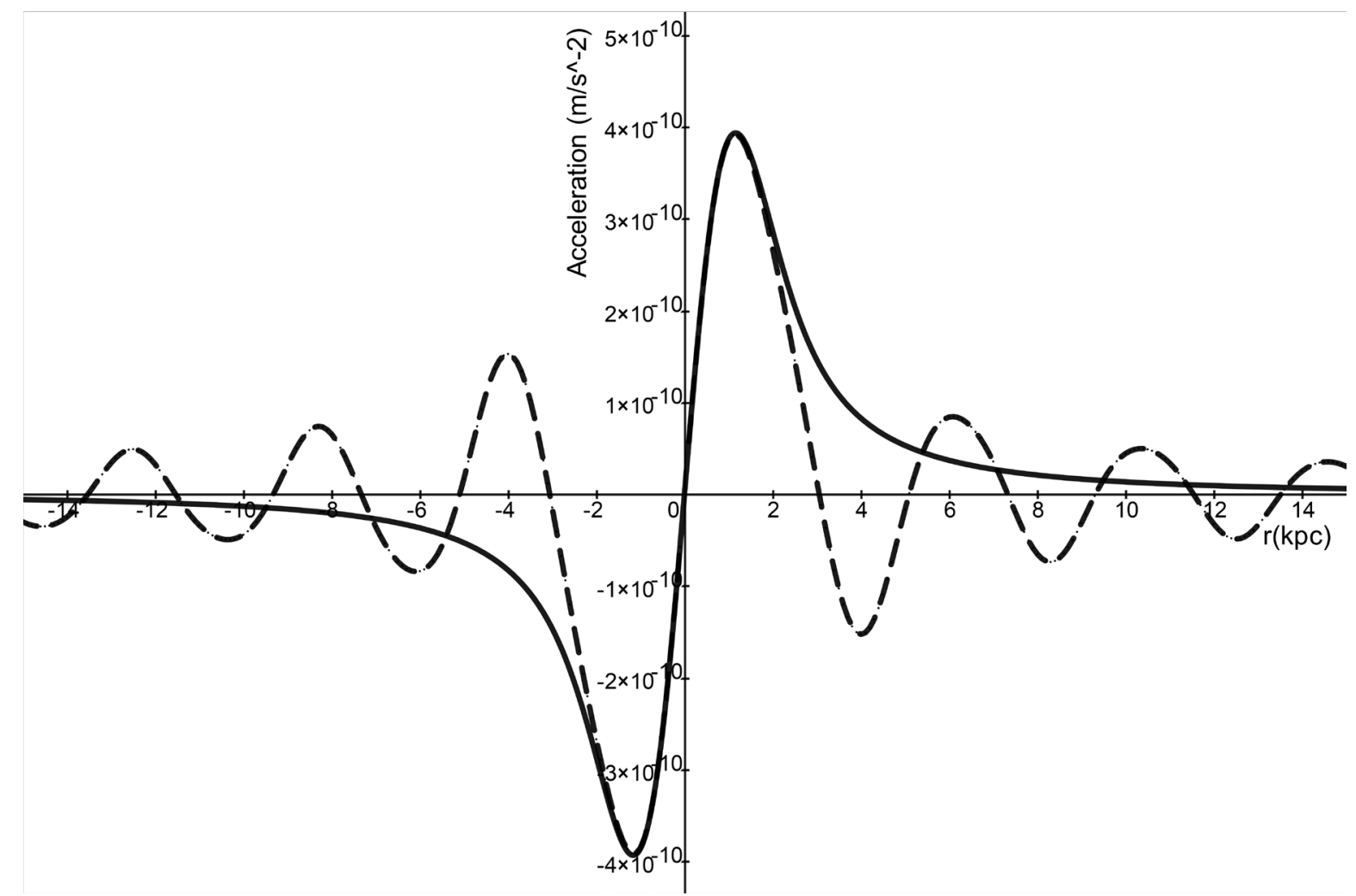

Figure 6. Force per unit mass (acceleration) in the classical Newtonian (solid line) and new proposed model (dashed line) with $k_{m}=1.5 \mathrm{kpc}^{-1}$. The point line shows that the resulting force is inscribed in a $1 / r$ envelope (points).

Being therefore sufficient the occurrence of these regions to explain the fact that most spiral galaxies have flat rotational velocity curves, since such galaxies have a high concentration of mass in a volume in their center (bulge) as modeled in the present case. Besides, there are adjacent regions of negative gravitational force, which effect is to expel objects orbiting the galaxy towards regions of positive gravitational force, being therefore most likely responsible for the creation of voids in the galaxies, where there is a lower concentration of matter.

The present model is perfectly symmetrical, requiring more realistic models for the precise determination of where the matter will accumulate. More precisely, it is necessary and suggested by the author to run complex computational simulations to analyze the evolution of spiral galaxies, considering the quantization of the spatial frequencies.

Now applying the relationship $v_{r}=\sqrt{r \cdot a(r)}$, where $v_{r}$ is the rotational velocity, $a(r)$ is the acceleration, and $r$ is the radial distance, we obtain Figure 7 where the negative values (gravitational repulsion) are not considered.

From Figure 7, fringes of extra gravitational force are created around the central bulge, providing areas where the velocity of rotation is practically constant. These fringes are also probably responsible for the creation of galactic arms, where areas of lower matter density are those with repulsive gravitational force (negative velocity). Regardless, it is suggested again as confirmation of the present theory the execution of computer simulations of the evolution of galaxies, considering the 


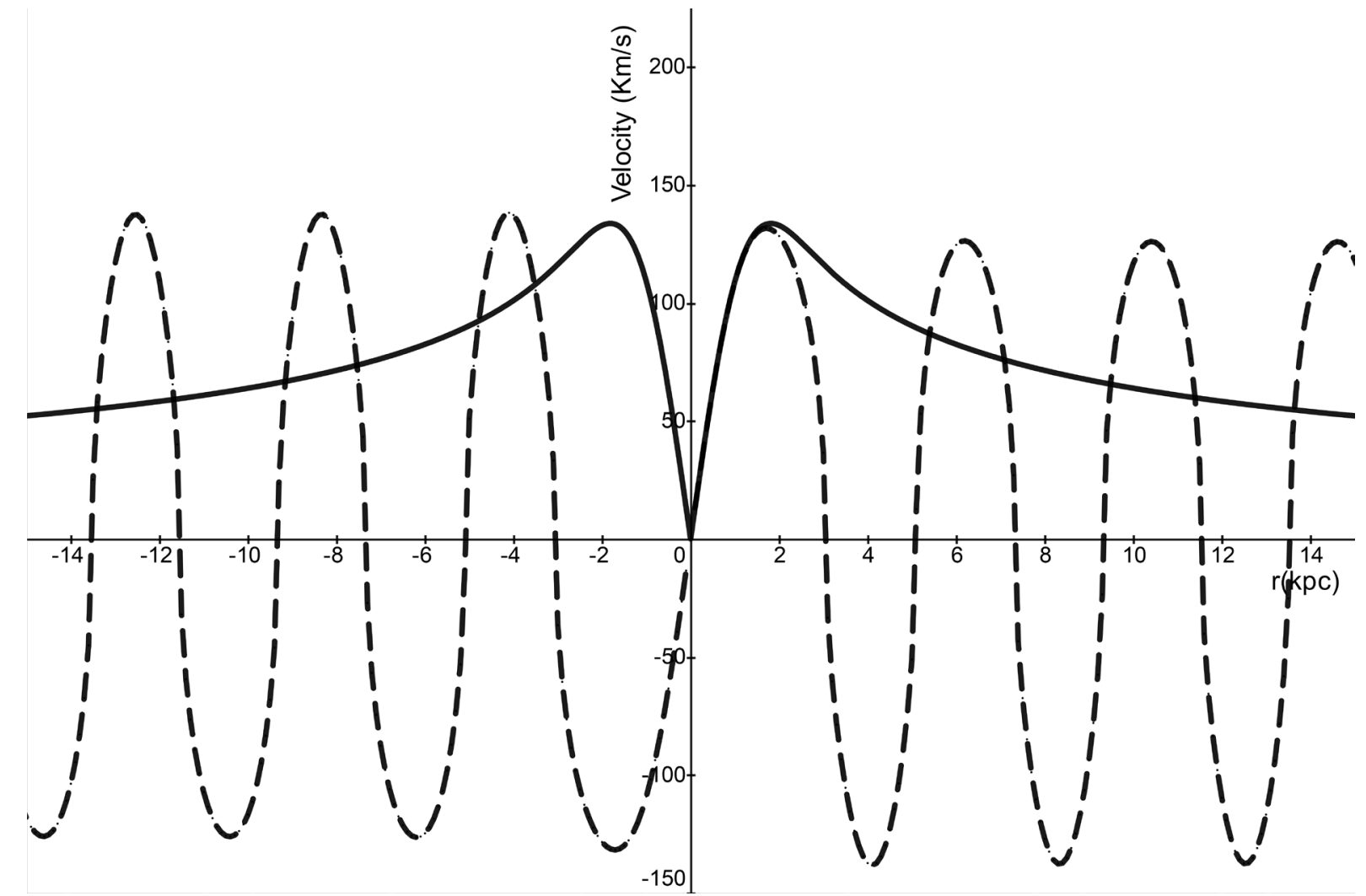

Figure 7. Rotational velocity using the relation $v_{r}=\sqrt{r \cdot a(r)}$ in the classical Newtonian way (solid line) and the new proposed model (dashed line) with $k_{m}=1 \mathrm{kpc}^{-1}$.

quantization of the spatial frequency $k$. The quantization of the spatial frequency used in Figure 6 and Figure 7 is $k_{m}=1 \mathrm{kpc}^{-1}$, which is not necessarily the exact value, but apparently close to the real value. Computer simulations must be performed in order to determine a more accurate value.

\subsection{Dark Energy}

Knowing that the acceleration $a_{c o}$ in terms of the displacement variable $s$, can be expressed as indicated in Equation (16).

$$
a_{c c}=\frac{\mathrm{d} v}{\mathrm{~d} t}=\frac{\mathrm{d} v}{\mathrm{~d} s} \frac{\mathrm{d} s}{\mathrm{~d} t}=\frac{\mathrm{d} v}{\mathrm{~d} s} \cdot v \leftrightarrow a_{c c} \cdot \mathrm{d} s=v \cdot \mathrm{d} v
$$

Integrating both sides and solving for the velocity $v$, we obtain

$$
\int a_{c c} \cdot \mathrm{d} s=\int v \cdot \mathrm{d} v \leftrightarrow v=\sqrt{2 \int a_{c c}(s) \cdot \mathrm{d} s}
$$

Therefore, we have an expression that relates the variations of acceleration in space $s$, with velocity v acquired by a mass. Consequently, we notice that if the integral of the acceleration in space is limited $\left(\int a_{c c}(s) \cdot \mathrm{d} s\right)$, the velocity $v$ will have a constant value, causing a zero acceleration of a mass placed in that field. Now considering $a(r)$ as this acceleration, we have the necessary conditions to verify if the acceleration of gravity with the effect of the quantization of the spa- 
tial frequency causes a net acceleration or not. The force per unit mass a(r) (acceleration) due to the quantization varies positively and negatively inscribed in an envelope of $1 / r$ (Figure 6). Thus, it can be proved by integral convergence rules, that the velocity acquired by a mass placed in this gravitational field does not exhibit any net acceleration.

According to what was discussed above, we must assume that, on average, the net gravitational force between galaxies is canceled. It hence requires a force of repulsion much smaller than what is currently used to explain the accelerating expansion of the universe.

The next topic goes on to explain that the average luminous density of the universe is a sufficient factor to explain the commonly accepted rates of acceleration of the universe.

\section{Luminosity as the Factor for Universe Expansion}

In this topic, the influence of luminosity on the expansion of the universe is evaluated considering the net gravitational effect of the attraction between galaxies as null, as previously discussed.

Luminosity is defined as the total electromagnetic power emitted by a source, and for astronomy, such sources can be stars, galaxies, supernovae, or any other astronomical object [20]. The unity of luminosity is $W$, and this power is due to photons that individually carry a discrete amount of energy given by $h f$. Typically, an astronomical object such as a star emits its luminosity in a broad range of frequencies (bolometric luminosity). Each photon is associated with a momentum given by $h f / c$, where $c$ is the speed of light in the vacuum. It means that a stream of photons emitted by a source can exert a force over an object, being this force proportional to the cross-section area $S$ of the object which lies perpendicular to the direction of the stream of photons. For an object apart from the light source, the power per square meter $\left(\mathrm{W} / \mathrm{m}^{2}\right)$, commonly named "apparent brightness," is proportional to the inverse of the square of the distance $\left(1 / r^{2}\right)$. This relation is quite useful for our study, since we can borrow some concepts used in classical gravity, which also follow an inverse square law.

Considering that the universe is homogeneous and isotropic when viewed at large scales, we can make use of the shell theorem, which allows significant simplifications for objects subjected to gravitational forces inside or outside a shell or sphere.

We realize, in Figure 8, with the application of the shell theorem, that the entire universe outside the sphere does not exert any gravitational force over the astronomical object $A$; and all the gravitational effect of the objects inside the sphere over the object $A$ can be replaced by an equivalent object in the center of the sphere with a mass equivalent to the sum of all masses within the sphere.

Since apparent brightness $\left(\mathrm{W} / \mathrm{m}^{2}\right)$ also follows the inverse square law, just like the force of gravity, we can, by analogy, use the same simplification as for the luminosity. 


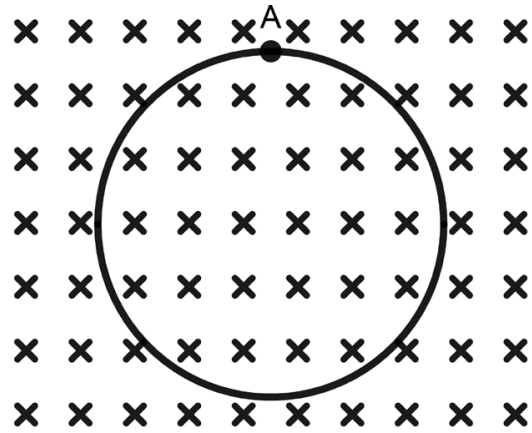

(a)

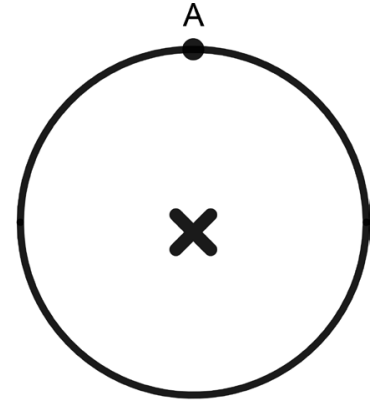

(b)

Figure 8. Shell theorem for gravity applied for a homogeneous and isotropic universe (a), and the equivalent gravitational effect in (b). This simplification is also valid for the "luminosity push".

Using again Figure 8, we can also state that the equivalent "luminosity push" felt by an object $A$ in Figure 8(a) is derived not taking into account all the luminosities sources outside the sphere, but replacing all the luminosity sources inside the sphere by an equivalent one at the center with a luminosity equivalent to the sum of all luminosities inside the sphere (Figure 8(b)).

This simplification, again, is only possible because, just like gravity, the apparent brightness also follows the inverse square law $\left(1 / r^{2}\right)$. However, this simplification has some constraints; among them, we can list:

1) The presence of intergalactic dust causes an attenuation of the order $0.01 \mathrm{mag} \cdot \mathrm{h} \cdot \mathrm{Gpc}^{-1}$ [21], being negligible for a radius $R<1 \mathrm{Gpc}$.

2) The mean free path M.F.P for a photon traveling through the local universe is given by Equation (18), Where $R_{g}$ is the mean galaxy radius, and $n$ is mean density of galaxies per volume. $n$ can be estimated as $0.014 \mathrm{~h}^{3} \cdot \mathrm{Mpc}^{-3}$ [22], and $R_{g}$ as $0.015 \mathrm{Mpc}$. Replacing these values into Equation (18) we obtain M.P.F $=101.05$ $\mathrm{h}^{-1} \mathrm{Gpc}$.

$$
M . F . P=\frac{1}{n \cdot \pi \cdot R_{g}^{2}}
$$

So, assuming the two constraints above, we can fairly say the universe is transparent for our analysis, being this way, the simplification given by Figure 8 is valid.

\subsection{Derivation of the Hubble Parameter}

Since the universe is expanding, the mean luminosity density given by $\rho_{L}(t)$ varies in time. It leads to a total luminosity $L$ inside a sphere of a comoving radius $R(t)$ given by:

$$
L=\rho_{L}(t) \cdot \frac{4 \cdot \pi \cdot R(t)^{3}}{3}
$$

Generating a luminosity pressure $P$

$$
P=\frac{L}{4 \pi R^{2}} \frac{1}{C}
$$


According to [23], the Tolman relation, which expresses the redshift dependence of the surface's bolometric luminosities of the galaxies $B$, is

$$
B(z)=(1+z)^{-4} B_{0}
$$

where $z$ is the redshift, and $B_{0}$ is the surface's bolometric luminosity at $z=0$. The Tolman relation implies that galaxies at redshift $z$ will appear fainter by a factor of $(1+z)^{4}$. This relation is valid for an observer placed at $z=0$. If the observer is moved to a position at the point $A$, at a redshift $z$, in relation to the center of a sphere, as indicated in Figure 8, we must reverse this relation, indicating that in the past, the surface brightness of the galaxies was brighter. Knowing that, and that the scale factor $a$ and the redshift are related by $a=(1+z)^{-1}$, the luminosity pressure, as indicated in Equation (20), should be modified by

$$
P=\frac{1}{a^{4}} \frac{L}{4 \pi R^{2}} \frac{1}{c}
$$

A galaxy with a face-on surface $S$, as indicated in Figure 9, opacity $k$ and mass $M$, is subject to an acceleration given by

$$
A_{c c}=\frac{1}{a^{4}} \cdot \frac{L}{4 \pi R^{2}} \cdot \frac{1}{C} \cdot \frac{S \cdot k}{M}
$$

Replacing $L$ of Equation (19) in Equation (22), produces

$$
A_{c c}=\frac{1}{a^{4}} \cdot \frac{\rho_{L}(t) \cdot R(t)}{3} \cdot \frac{1}{c} \cdot \frac{S \cdot k}{M}
$$

Applying the scale factor $a(t) \cdot R_{0}=R(t)$, we get the following relationship for acceleration

$$
A_{c c}=\frac{\mathrm{d}^{2} a(t)}{\mathrm{d} t^{2}}=\ddot{a} \cdot R\left(t_{1}\right)
$$

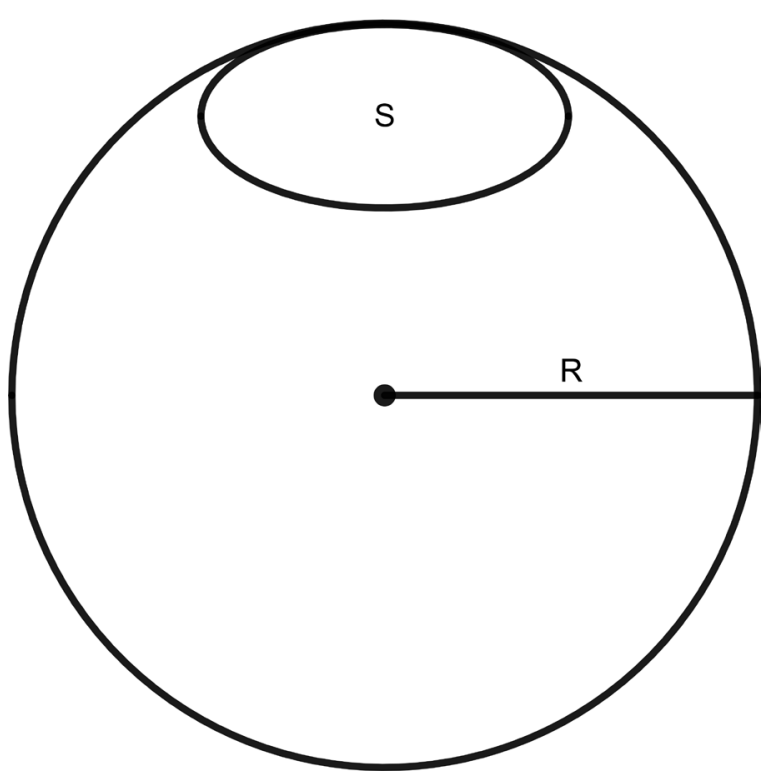

Figure 9. Galaxy with Area $S$, opacity $K$ and mass $M$ in the surface of a sphere of radius $R$. 
The Equation (25) is derived in [24].

$$
\rho_{L}(t)=\frac{\rho_{L}\left(t_{1}\right)}{a^{3}}
$$

Where $t_{1}$ is the present time and $\rho_{L}\left(t_{1}\right)$ is the luminosity density of the present time. Replacing Equations (24) and (25) in Equation (23) yield:

$$
\left.\begin{array}{l}
\ddot{a} \cdot R\left(t_{1}\right)=\frac{1}{a^{4}} \cdot \frac{\rho_{L}\left(t_{1}\right) \cdot a^{3} \cdot R\left(t_{1}\right)}{a^{3} \cdot 3} \cdot \frac{1}{c} \cdot \frac{S \cdot k}{M}=\frac{1}{a^{4}} \cdot \frac{\rho_{L}\left(t_{1}\right) \cdot R\left(t_{1}\right)}{3} \cdot \frac{1}{c} \cdot \frac{S \cdot k}{M} \\
\ddot{a}=\frac{1}{a^{4}} \cdot \frac{\rho_{L}\left(t_{1}\right)}{3} \cdot \frac{1}{c} \cdot \frac{S \cdot k}{M}
\end{array}\right\}
$$

The Equation (26) is correct for galaxies, which are partially opaque [25]. In general, its opacity depends on the wavelength and topology of the galaxies. Most of the opacity of galaxies is related to absorption and scattering of photons, and it is directly related to its dust content, occurring mostly for higher frequencies in the UV-Blue range of wavelengths. Extensive studies showing such relations are found in [26] [27].

Studies conducted by [21] placed the opacity $K$ of spiral galaxies in the range $0.48 \pm 0.15$. Integrating Equation (26) in $a$ and dividing by $a^{2}$, produces

$$
\left(\frac{\dot{a}}{a}\right)^{2}=-\frac{2 \cdot \rho_{L}\left(t_{1}\right) \cdot S \cdot k}{a^{5} \cdot 9 \cdot c \cdot M}+\frac{2 \cdot B}{a^{2}}
$$

Which is an expression for the Hubble constant since $H=\dot{a} / a$, where $B$ is a term to be determined. The term $B$ can be determined using initial conditions, assuming that the gravitational force between galaxies is on average, nullified. This means that for galaxies, placed, as shown in Figure 10, the luminosity pressure is dominant, where the scale factor is $\approx 2 R_{g}$, with $R_{g}$ being the average galactic radius. We can call the process, where the galaxies start to move away as "luminosity dominance," and the time of its occurrence as $t_{D}$, during this time we have

$$
a\left(t_{D}\right)=\frac{R\left(t_{D}\right)}{R\left(t_{1}\right)}
$$

where $R\left(t_{D}\right)$ is the mean distance between galaxies at the "luminosity dominance" time $t_{d}$, and $R\left(t_{1}\right)$ is the mean distance between galaxies at the present

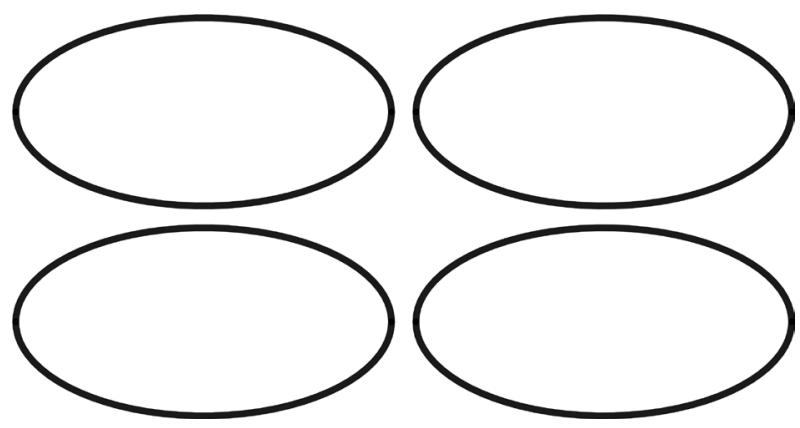

Figure 10. Galaxies apart in 2. $R_{g}$ at time $t_{D}$, when the pressure of light begins to dominate over the gravitational force. 
time $t_{1}$.

The mean distance between galaxies at time $t_{D}$, as seen in Figure 10, is approximately twice as the galactic radius, being the average galaxy radius commonly assumed to be $10 \mathrm{kpc}$ [28] [23]. Then, $R\left(t_{D}\right)=2 \cdot R_{g}=20 \mathrm{kpc}$, and the present time average distance between galaxies can be derived using [29].

$$
R\left(t_{1}\right)=\left(\frac{3}{4 \pi n}\right)^{\frac{1}{3}}
$$

where $n$ is the present time density number of galaxies. Another important consideration to be made is assuming that at the time of the "luminosity dominance" $t_{D}$ the recessional velocity is zero since it is the time when the luminosity repulsive force starts to overcome the gravitational force. So, consequently

$$
\dot{a}\left(t_{D}\right)=0
$$

Applying Equations (28) and (30) in Equation (27), we obtain

$$
\left(\frac{\dot{a}\left(t_{D}\right)}{a\left(t_{D}\right)}\right)^{2}=0=-\frac{2 \cdot \rho_{L}\left(t_{1}\right) \cdot S \cdot k}{a\left(t_{D}\right)^{5} \cdot 9 \cdot c \cdot M}+\frac{2 \cdot B}{a\left(t_{D}\right)^{2}}
$$

Solving for $B$ yields

$$
B=\frac{\rho_{L}\left(t_{1}\right) \cdot S \cdot k}{a\left(t_{D}\right)^{3} \cdot 9 \cdot c \cdot M}
$$

Replacing this in Equation (27) we obtain

$$
\left(\frac{\dot{a}}{a}\right)^{2}=-\frac{2 \cdot \rho_{L}\left(t_{1}\right) \cdot S \cdot k}{a^{5} \cdot 9 \cdot c \cdot M}+\frac{2 \cdot \rho_{L}\left(t_{1}\right) \cdot S \cdot k}{a\left(t_{D}\right)^{3} \cdot a^{2} \cdot 9 \cdot c \cdot M}
$$

Rearranging for the Hubble constant

$$
H(t)=\sqrt{-\frac{2 \cdot \rho_{L}\left(t_{1}\right) \cdot S \cdot k}{a(t)^{5} \cdot 9 \cdot c \cdot M}+\frac{2 \cdot \rho_{L}\left(t_{1}\right) \cdot S \cdot k}{a\left(t_{D}\right)^{3} \cdot a(t)^{2} \cdot 9 \cdot c \cdot M}}
$$

\subsection{Derivation of the Scale Factor A}

OBS: The factor scale factor a here presented is not the acceleration.

Considering the Equation derived for the Hubble constant (Equation (34)), we can verify that the second term inside the square root is equal to the first term when $t=t_{D}$. For $t>t_{D}$ the first term increasingly dominates as the time increases. So, for $t \gg t_{D}$ we can approximate the Equation (34) as

$$
H(t)=\frac{\dot{a}}{a}=\sqrt{\frac{2 \cdot \rho_{L}\left(t_{1}\right) \cdot S \cdot k}{a\left(t_{D}\right)^{3} \cdot a(t)^{2} \cdot 9 \cdot c \cdot M}}
$$

Rearranging we obtain

$$
\dot{a}^{2}=\frac{2 \cdot \rho_{L}\left(t_{1}\right) \cdot S \cdot k}{a\left(t_{D}\right)^{3} \cdot 9 \cdot c \cdot M}
$$

Equation (36) can be solved for the scale factor a. We can "guess" a solution 
form for the scale factor $a$ as being

$$
a(t)=D \cdot t^{U}
$$

where $t$ is time, $D$ and $U$ are constants to be determined, replacing Equation (37) in Equation (36) and rearranging we get

$$
D^{2} \cdot U^{2} \cdot t^{2 U-2}=\frac{2 \cdot \rho_{L}\left(t_{1}\right) \cdot S \cdot k}{a\left(t_{D}\right)^{3} \cdot 9 \cdot c \cdot M}
$$

Since the right term is a constant, we need to set $U$ to force the left term to be also a constant, this is done doing

$$
2 U-2=0 \rightarrow U=1
$$

Replacing $U=1$ in Equation (38) and solving for $D$ yields

$$
D=\sqrt{\frac{2 \cdot \rho_{L}\left(t_{1}\right) \cdot S \cdot k}{a\left(t_{D}\right)^{3} \cdot 9 \cdot c \cdot M}}
$$

Therefore, the approximated scale factor for $t \gg t_{D}$ is

$$
a(t) \approx \sqrt{\frac{2 \cdot \rho_{L}\left(t_{1}\right) \cdot S \cdot k}{a\left(t_{D}\right)^{3} \cdot 9 \cdot c \cdot M}} \cdot t
$$

The Equation (41) is a good approximation for values of $t \gg t_{D}$ but is not the whole answer for $a(t)$, since if we derive twice the value present in Equation (41) it will become zero, what is not valid, since the acceleration value for $\ddot{a}(t)$ is given in Equation (37). Therefore, we can refine the value of $a(t)$ doing the same procedure as before for the acceleration term in Equation (37). Calling the acceleration term as $a_{a c c}(t)$, and guessing the answer to be in the form $a_{a c c}(t)=D \cdot t^{U}$, we get

$$
D^{5} \cdot U \cdot(U-1) \cdot t^{5 \cdot U-2}=\frac{\rho_{L}\left(t_{1}\right)}{3} \cdot \frac{1}{c} \cdot \frac{S \cdot k}{M}
$$

Using the same procedure as in Equation (39) leads to $U=5 / 2$, and solving for $D$ produces

$$
a_{a c c}(t)=-\left(\frac{25 \cdot \rho_{L}\left(t_{1}\right) \cdot S \cdot k}{18 \cdot c \cdot M}\right)^{\frac{1}{5}} \cdot t^{\frac{2}{5}}
$$

Adding this term to Equation (41), yields

$$
a(t) \approx-\left(\frac{25 \cdot \rho_{L}\left(t_{1}\right) \cdot S \cdot k}{18 \cdot c \cdot M}\right)^{\frac{1}{5}} \cdot t^{\frac{2}{5}}+\sqrt{\frac{2 \cdot \rho_{L}\left(t_{1}\right) \cdot S \cdot k}{a\left(t_{D}\right)^{3} \cdot 9 \cdot c \cdot M}} \cdot t
$$

Considering our initial time as $t_{D}=0$, we have the value of $a\left(t_{D}\right)$ in such a case. Adding this to Equation (44) we obtain the complete solution for the scale factor as a function of time $t$ expressed in Equation (45).

$$
a(t)=-\left(\frac{25 \cdot \rho_{L}\left(t_{1}\right) \cdot S \cdot k}{18 \cdot c \cdot M}\right)^{\frac{1}{5}} \cdot t^{\frac{2}{5}}+\sqrt{\frac{2 \cdot \rho_{L}\left(t_{1}\right) \cdot S \cdot k}{a\left(t_{D}\right)^{3} \cdot 9 \cdot c \cdot M}} \cdot t+a\left(t_{D}\right)
$$




\subsection{Results of Acceleration for the Milky Way}

According to [22], the luminosity density at the present time $\rho_{L}\left(t_{1}\right)$ is estimated in $\approx(2.6 \pm 0.3) \times 10^{8} \mathrm{~h} \cdot \mathrm{L}_{\odot} \cdot \mathrm{Mpc}^{-3}$ and the number density of galaxies is estimated to be $n \approx(1.46 \pm 0.12) \times 10^{-2} \mathrm{~h}^{3} \cdot \mathrm{Mpc}^{-3}$. Using the Equation (29) with $n=1.46 \times 10^{-2}$, we obtain $R\left(t_{1}\right)=2.538 \mathrm{Mpc}$. Therefore, considering $R\left(t_{D}\right)=20 \mathrm{kpc}$, we get

$$
a\left(t_{D}\right)=\frac{R\left(t_{D}\right)}{R\left(t_{1}\right)}=\frac{20 \mathrm{kpc}}{2.538 \mathrm{Mpc}}=7.88 \times 10^{-3}
$$

[30] have reported that the mass of the Milky Way galaxy is 1.5 trillion solar masses, being about $90 \%$ of this mass dark matter. So, the visible, baryonic mass is about 0.15 trillion solar masses. The present work uses only the visible matter for the calculation, since as reported in the present work, the extra dark matter is only consequence of the effect of the quantization of the spatial frequency, not being accounted as inertial mass.

[30] also reported a Milky Way radius of about $39 \mathrm{kpc}$, over twice as much as previously reported, being most of this extra part due to the globular cluster out in the galactic halo. Therefore, for the calculation, only the disc radius is considered, being about $15 \mathrm{kpc}$ [31], resulting in a face-on area of about $148 \mathrm{kpc}^{2}$. For the opacity, $k$ is considered the values obtained by [21] being about 0.48 for spiral galaxies. So, in resume, we have the following parameters, for the Milky Way galaxy, in SI base units:

$$
\begin{gathered}
\rho_{L}\left(t_{1}\right)=3.387 \times 10^{-33} \mathrm{~W} \cdot \mathrm{m}^{-3} \\
S=6.734 \times 10^{41} \mathrm{~m}^{2} \\
k=0.48 \\
M=3 \times 10^{41} \mathrm{~kg} \\
c=3 \times 10^{8} \mathrm{~m} \cdot \mathrm{s}^{-1} \\
a\left(t_{D}\right)=7.88 \times 10^{-3}
\end{gathered}
$$

Applying those values for Equation (34), for the present time $t_{1}$, with $a\left(t_{1}\right)=1$, we obtain

$$
H\left(t_{1}\right)=\frac{2.35 \times 10^{-18}}{\mathrm{~s}}=72.54 \mathrm{~km} \cdot \mathrm{s}^{-1} \cdot \mathrm{Mpc}^{-1}
$$

which agrees very well within the interval of acceptable values for the Hubble constant $\approx 67-73 \mathrm{~km} \cdot \mathrm{s}^{-1} \cdot \mathrm{Mpc}^{-1}$, obtained respectively by [12] [13].

Using Equation 34, we can also plot the normalized value of the Hubble parameter $\left(H(a) / H\left(t_{1}\right)\right)$ against the scale factor $a$, with the result indicated in Figure 11.

Using the Equations (34) and (45) we can also plot the normalized value of the Hubble parameter $\left(H(t) / H\left(t_{1}\right)\right)$ and scale factor a against time in years (Yr), with the result indicated in Figure 12. 


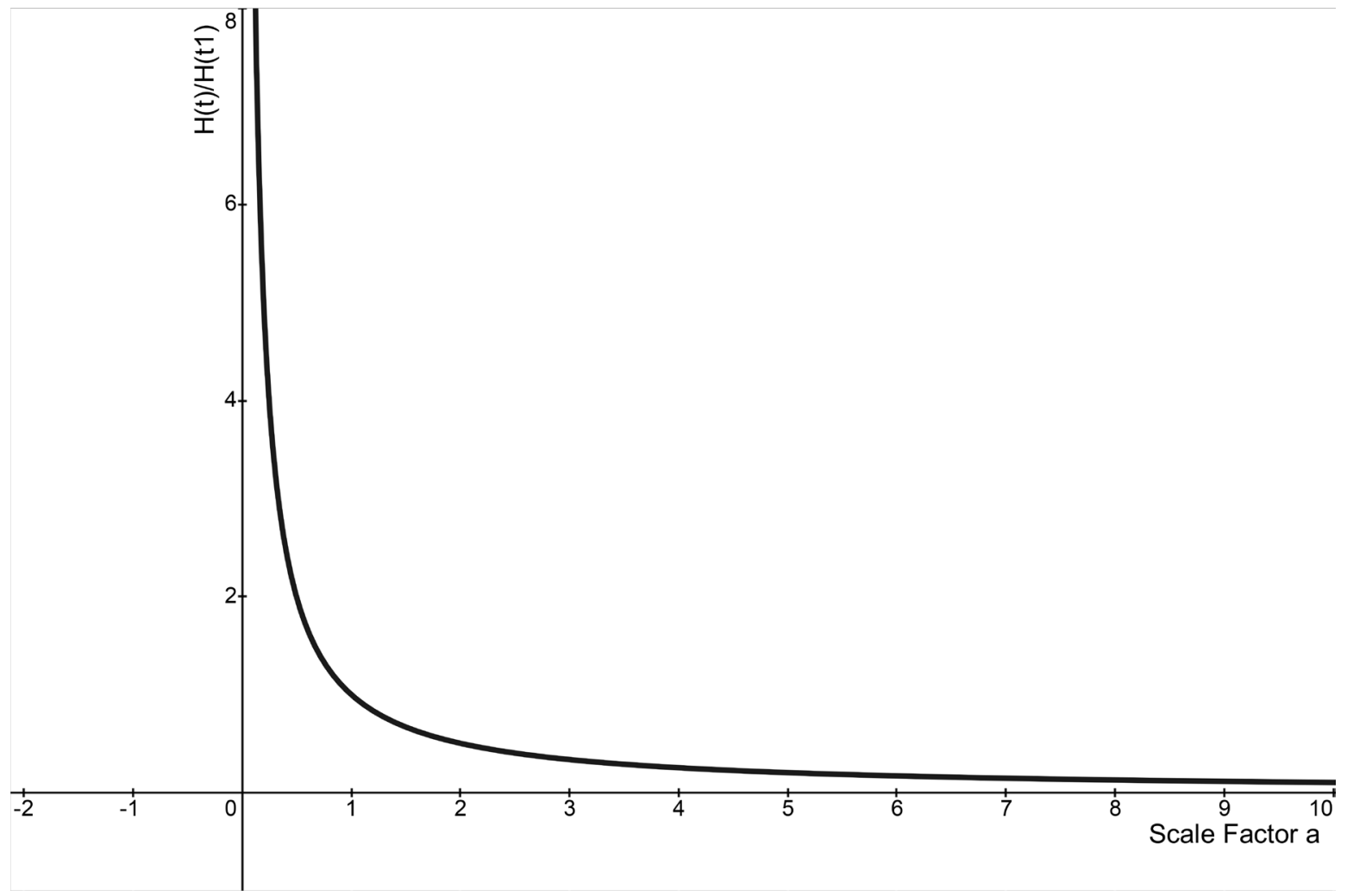

Figure 11. Normalized value of the Hubble parameter $H(a) / H\left(t_{1}\right)$ plotted against the scale factor $a$.

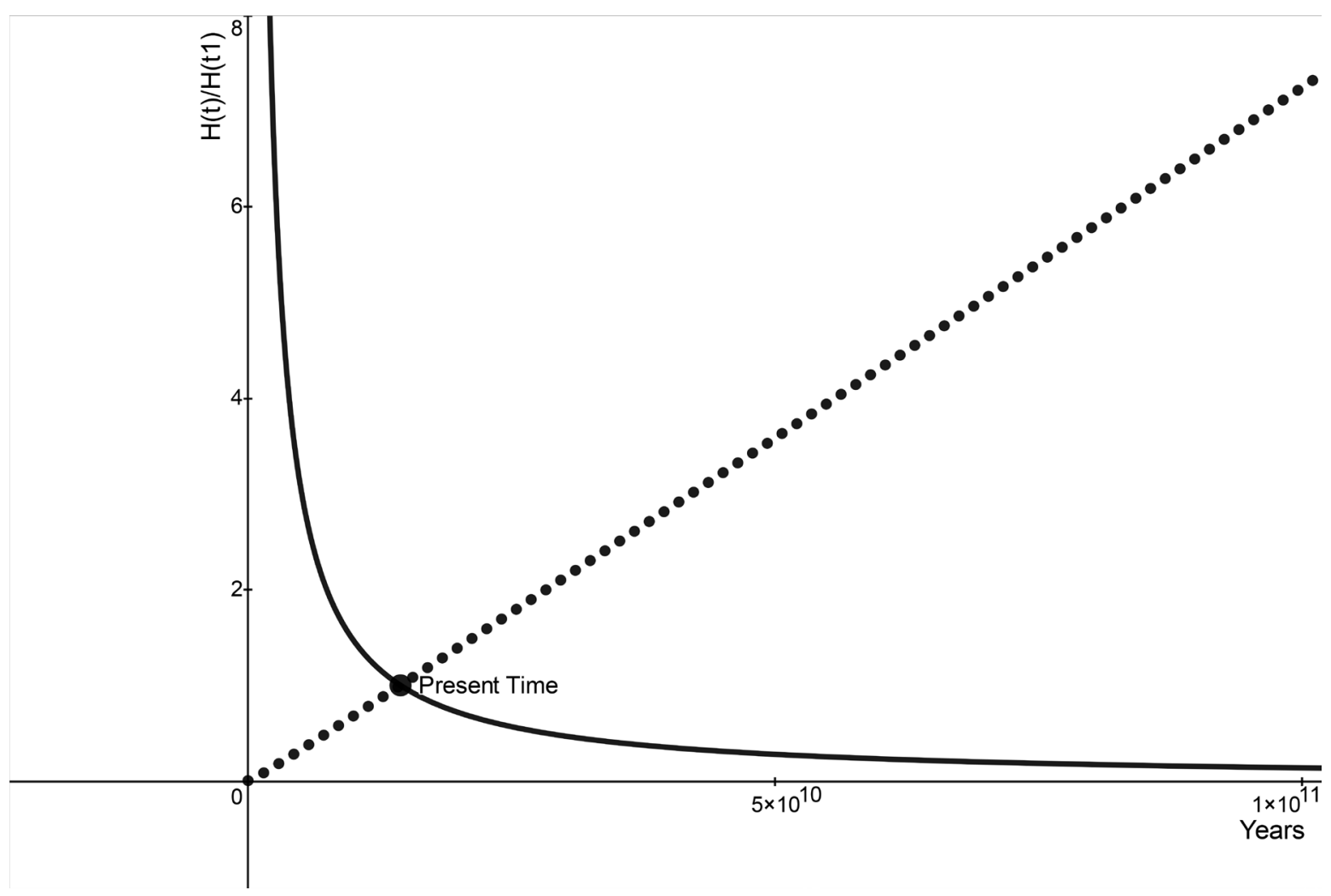

Figure 12. Normalized value of the Hubble parameter $\left(H(t) / H\left(t_{1}\right)\right)$ in solid line and scale factor (point line) plotted against time in years. 
Looking at Figure 12, we realize that the universe is coasting type, and thus will expand steadily and never contracts. Analyzing the Equation (35), we realize that the only term that varies from one galaxy to another is the expression S.K/M. As the Hubble constant is approximately the same for a wide range of galaxy types, not depending on their inclination too, we are led to conclude that the term $S . K / M$ is approximately constant for all galaxies and remains approximately constant no matter the inclination. Analyzing in a simple way, we conclude that this could be the case since for inclined galaxies, the cross-section area $S$ is reduced, and since the photons have to cross more matter, intuitively, the opacity $K$ will grow at the same proportion, keeping the term S.K approximately constant.

\section{Discussion}

The developed theory proved to be entirely satisfactory in developing a model of the gravitational force for massive objects, such as non-rotating black holes, in line with Einstein's theory of relativity $(G R)$. As merit, the present theory is able to determine the behavior of the acceleration of gravity within a black hole. Besides, the theory offers a straightforward and simple method to numerically calculate the effect of gravity near massive objects. It also provides a "bridge" between gravity and quantum physics, since the deflection of the space-time fabric can be understood as originated in the quantum scale.

The developed theory also proved to be quite satisfactory in the development of a model for the dark matter, besides demonstrating the average absence of gravitational force between galaxies. From the absence of average force between galaxies, it was possible to demonstrate that the average luminosity density of the universe is enough to explain the currently accepted rates of expansion of the universe. As a suggestion for future studies, the author recommends simulations of evolution of the galaxies, taking into consideration the effect of quantization of the spatial frequency $k$.

\section{Conflicts of Interest}

The author declares no conflicts of interest regarding the publication of this paper.

\section{References}

[1] Einstein, A. (1915) Die feldgleichungen der gravitation. Sitzung der Physikalisch-Mathematischen Klasse, 25, 844-847.

[2] Brustein, R. and Medved, A.J.M. (2019) Resisting Collapse: How Matter inside a Black Hole Can Withstand Gravity. Physical Review D, 99, Article ID: 064019. https://doi.org/10.1103/PhysRevD.99.064019

[3] Baumgarte, T.W., Shapiro, S.L. and Pullin, J. (2011) Numerical Relativity: Solving Einstein's Equations on the Computer. Physics Today, 64, 49.

[4] Maccone, L. (2019) A Fundamental Problem in Quantizing General Relativity. Foundations of Physics, 49, 1394-1403. https://doi.org/10.1007/s10701-019-00311-w 
[5] Bera, R.K. (2020) Waves and Fourier Analyses. In: The Amazing World of Quantum Computing, Springer, Singapore, 99-110. https://doi.org/10.1007/978-981-15-2471-4 5

[6] Perlov, D. and Vilenkin, A. (2017) Cosmology for the Curious. Springer, Berlin. https://doi.org/10.1007/978-3-319-57040-2

[7] Wang, B., Abdalla, E., Atrio-Barandela, F. and Pavón, D. (2016) Dark Matter and Dark Energy Interactions: Theoretical Challenges, Cosmological Implications and Observational Signatures. Reports on Progress in Physics, 79, Article ID: 096901. https://doi.org/10.1088/0034-4885/79/9/096901

[8] Wilhelm, K. and Dwivedi, B.N. (2020) On the Radial Acceleration of Disc Galaxies. Monthly Notices of the Royal Astronomical Society, 494, 4015-4025.

https://doi.org/10.1093/mnras/staa967

[9] Riess, A.G., Filippenko, A.V., Challis, P., Clocchiatti, A., Diercks, A., Garnavich, P.M., Gilliland, R.L., Hogan, C.J., Jha, S., Kirshner, R.P. and Leibundgut, B.R.U.N.O. (1998) Observational Evidence from Supernovae for an Accelerating Universe and a Cosmological Constant. The Astronomical Journal, 116, 1009.

https://doi.org/10.1086/300499

[10] Perlmutter, S., Aldering, G., Goldhaber, G., Knop, R.A., Nugent, P., Castro, P.G., Deustua, S., Fabbro, S., Goobar, A., Groom, D.E. and Hook, I.M. (1999) Measurements of $\Omega$ and $\Lambda$ from 42 High-Redshift Supernovae. The Astrophysical Journal, 517, 565. https://doi.org/10.1086/307221

[11] Schweitz, J.Å. (2020) Expansion of the Universe: Back to Basics.

[12] Bennett, C.L., Larson, D., Weiland, J.L. and Hinshaw, G. (2014) The 1\% Concordance Hubble Constant. The Astrophysical Journal, 794, 135. https://doi.org/10.1088/0004-637X/794/2/135

[13] Freedman, W.L., Madore, B.F., Gibson, B.K., Ferrarese, L., Kelson, D.D., Sakai, S., Mould, J.R., Kennicutt Jr., R.C., Ford, H.C., Graham, J.A. and Huchra, J.P. (2001) Final Results from the Hubble Space Telescope Key Project to Measure the Hubble Constant. The Astrophysical Journal, 553, 47. https://doi.org/10.1086/320638

[14] Estrada, R. (2014) On Radial Functions and Distributions and Their Fourier Transforms. Journal of Fourier Analysis and Applications, 20, 301-320. https://doi.org/10.1007/s00041-013-9313-2

[15] Einstein, A. (2019) Relativity: The Special and the General Theory-100th Anniversary Edition. Princeton University Press, Princeton.

https://doi.org/10.2307/j.ctv69tgs1

[16] Phillips, A.C. (2013) Introduction to Quantum Mechanics. John Wiley \& Sons, Hoboken.

[17] Rindler, W. (2006) Relativity-Special, General, and Cosmological. Oxford University Press, New York.

[18] Miyamoto, M. and Nagai, R. (1975) Three-Dimensional Models for the Distribution of Mass in Galaxies. Publications of the Astronomical Society of Japan, 27, 533-543.

[19] Higgins, J.R. (1996) Sampling Theory in Fourier and Signal Analysis: Foundations. Oxford University Press on Demand, Oxford.

[20] Morison, I. (2013) Introduction to Astronomy and Cosmology. John Wiley \& Sons, Hoboken.

[21] Vavryčuk, V. (2016) Universe Opacity and EBL. Monthly Notices of the Royal Astronomical Society, 465, 1532-1542. https://doi.org/10.1093/mnras/stw2825

[22] Blanton, M.R., Dalcanton, J., Eisenstein, D., Loveday, J., Strauss, M.A., SubbaRao, 
M., Weinberg, D.H., Anderson Jr, J.E., Annis, J., Bahcall, N.A. and Bernardi, M. (2001) The Luminosity Function of Galaxies in SDSS Commissioning Data. The Astronomical Journal, 121, 2358-2380. https://doi.org/10.1086/320405

[23] Reynolds, A.P., Parmar, A.N., Hakala, P.J., Pollock, A.M.T., Williams, O.R., Peacock, A. and Taylor, B.G. (1999) The EXOSAT Medium-Energy Slew Survey Catalog. Astronomy and Astrophysics Supplement Series, 134, 287-300.

https://doi.org/10.1051/aas:1999140

[24] Jaffe, A.H. (2012) Cosmology 2012: Lecture Notes. Imperial College, London.

[25] White III, R.E., Keel, W.C. and Conselice, C.J. (2000) Seeing Galaxies through Thick and Thin. I. Optical Opacity Measures in Overlapping Galaxies. The Astrophysical Journal, 542, 761-778. https://doi.org/10.1086/317011

[26] Calzetti, D. and Heckman, T.M. (1999) The Evolution of Dust Opacity in Galaxies. The Astrophysical Journal, 519, 27. https://doi.org/10.1086/307338

[27] Calzetti, D., Armus, L., Bohlin, R.C., Kinney, A.L., Koornneef, J. and Storchi-Bergmann, T. (2000) The Dust Content and Opacity of Actively Star-Forming Galaxies. The Astrophysical Journal, 533, 682-695. https://doi.org/10.1086/308692

[28] Peebles, P.J.E. and Ratra, B. (2003) The Cosmological Constant and Dark Energy. Reviews of Modern Physics, 75, 559-606. https://doi.org/10.1103/RevModPhys.75.559

[29] Vavrycuk, V. (2019) Universe Opacity and Cosmic Dynamics.

[30] Starr, M. (2019) ScienceAlert.com.

[31] Rix, H.W. and Bovy, J. (2013) The Milky Way's Stellar Disk. The Astronomy and Astrophysics Review, 21, Article No. 61. https://doi.org/10.1007/s00159-013-0061-8 